\title{
An improved procedure for synthesis of some new 1,3-diaryl-2-propen-1-ones using PEG-400 as a recyclable solvent and their antimicrobial evaluation
}

\author{
BHASKAR S. DAWANE* \\ SHANKARAIAH G. KONDA* \\ BASEER M. SHAIKH \\ RAGHUNATH B. BHOSALE \\ Organic Research Laboratory \\ Department of Chemistry \\ Yeshwant Mahavidyalaya \\ Nanded-431602 (M.S) India
}

Accepted August 26, 2009

\begin{abstract}
A simple and convenient route is described for the synthesis of novel hetero 1,3-diaryl-2-propen-1-ones (chalcones) by using recyclable PEG-400 as an alternative reaction solvent. The reaction is clean with excellent yield, shorter reaction time and reduces the use of volatile organic compounds (VOCs). All the synthesized compounds were evaluated for their antimicrobial activities against several pathogenic representatives.
\end{abstract}

Keywords: 1,3-diaryl-2-propen-1-ones, PEG-400, antimicrobial activity

Chalcones (1,3-diaryl-2-propen-1-ones) constitute an important class of natural products belonging to the flavonoid family, which have been reported to possess a wide spectrum of biological activities, including antibacterial, antifungal, antiinflammatory, antitumor, insect antifeedant and antimutagenic (1-3). Additionally, some of chalcone derivatives have been found to inhibit several important enzymes in cellular systems, such as xanthine oxidase $(4)$ and protein tyrosine kinase $(5,6)$. Chalcones are also key precursors in the synthesis of many biologically important heterocycles such as benzothiazepine (7), pyrazolines (8), 1,4-diketones (9) and flavones (10). Hence, the synthesis of chalcones has generated vast interest among organic as well as medicinal chemists.

Reducing or eliminating the use of volatile organic solvents can minimize the generation of waste, which is a requirement of one of the principles of green chemistry (11, 12). Recently, poly etylene glycol (PEG) has been found to be an interesting solvent system. In continuation of own work on chalcones as precursors in the synthesis of various heterocycles (13), we have planned to synthesize a series of novel hetero chalcones by applying the principles of green chemistry, using PEG-400 as an alternative reaction medium (14). PEG is an environmentally benign reaction solvent, it is non-toxic, inexpensive, potentially recyclable and water soluble, which facilitates its removal from the reaction product.

\footnotetext{
*Correspondence; e-mail: bhaskardawane@rediffmail.com, kondasg@rediffmail.com
} 
B. S. Dawane et al.: An improved procedure for synthesis of some new 1,3-diaryl-2-propen-1-ones using PEG-400 as a recyclable solvent and their antimicrobial evaluation, Acta Pharm. 59 (2009) 473-482.

All the products (3a-p) were newly synthesized and characterized by their spectral analysis. Chemicals, $p$-chloro, $p$-amino and $p$-methoxy acetophenones were purchased from S. D. Fine Chemicals (India). Parent 5-chloro-2-hydroxy acetophenones, 5-methyl-2-hydroxy acetophenone and 5-chloro-4-methyl-2-hydroxy acetophenone were synthesized from $p$-chloro, $p$-methyl and 3-methyl-4-chloro phenols, respectively, by the Fries-reaction using acetic anhydride and $\mathrm{AlCl}_{3}$ (15). 2-Hydroxy-3-iodo substituted acetophenones were syntesized by our previously reported method (16). 2-Hydroxy-3-bromo substituted acetophenones were also synthesized by the bromination (bromine in acetic acid) method (15). 2-Butyl-4-chloro-5-formyl-imidazole was purchased from Sigma-Aldrich (India) and 2-amino-4-(4-chlorophenyl)-5-formyl-thiazole was prepared by the reported method (17).

\section{EXPERIMENTAL}

The title compounds were synthesized by Claisen-Schmidt condensation (15) using PEG-400 as reaction solvent. All the melting points were uncorrected and determined in an open capillary tube. The chemicals and solvents used were of laboratory grade and were purified. Completion of the reaction was monitored by thin layer chromatography on precoated sheets of silica gel-G (Merck, Germany) using iodine vapour for detection. IR spectra were recorded in $\mathrm{KBr}$ on a Shimadzu spectrometer (Japan). ${ }^{1} \mathrm{H}$ NMR spectra were recorded in DMSO- $d_{6}$ with an Avance spectrometer (Bruker, Germany) at $300-\mathrm{MHz}$ frequency using TMS as an internal standard. Mass spectra were recorded on an EI-Shimadzu QP 2010 PLUS GC-MS system (Shimadzu, Japan). Elemental analyses were performed on a Carlo Erba 106 Perkin-Elmer model 240 analyzer (Perkin-Elmer, USA).

The synthetic pathway is presented in Scheme 1 and physicochemical data for the synthesized compounds are given Table I.

General procedure for the synthesis of 1-(substituted phenyl), 3-(2-butyl-5-chloro-3H-imidazol-4-yl) or (2-amino-4-(4-chlorophenyl)-thiazol-5-yl)-2-propen-1-ones (3a-p)

An equimolar mixture of substituted acetophenone $\mathbf{1}(1 \mathrm{mmol})$, aromatic aldehyde 2 $(1 \mathrm{mmol})$ and $\mathrm{KOH}(2 \mathrm{mmol})$ was stirred in PEG-400 $(15 \mathrm{~mL})$ at $40{ }^{\circ} \mathrm{C}$ for 1 hour. After completion of the reaction (monitored by TLC), the crude mixture was worked up in ice-cold water $(100 \mathrm{~mL})$. The product which separated out was filtered. The filtrate was evaporated to remove water leaving PEG behind. The same PEG was utilized to synthesize further chalcones.

Spectroscopic data for all synthesized compounds are given in Table II.

\section{Antimicrobial activity}

All the synthesized compounds (3a-p) were screened for their in vitro antimicrobial activity by the agar diffusion method (18). Antibacterial activity was checked against the bacteria Escherichia coli, Salmonella typhi, Staphylococcus aureus and Bacillus subtillis. The culture strains of bacteria were maintained on a nutrient agar slant at $37 \pm 2{ }^{\circ} \mathrm{C}$ for 24-48 h. Antifungal activity was studied against Aspergillus niger, Aspergillus flavus, Peni- 
B. S. Dawane et al.: An improved procedure for synthesis of some new 1,3-diaryl-2-propen-1-ones using PEG-400 as a recyclable solvent and their antimicrobial evaluation, Acta Pharm. 59 (2009) 473-482.

Table I. Physical and analytical data of newly synthesized compounds

\begin{tabular}{|c|c|c|c|c|c|c|c|c|c|c|}
\hline \multirow{2}{*}{$\begin{array}{l}\text { Compd. } \\
\text { No. }\end{array}$} & \multirow{2}{*}{$\mathrm{R}_{1}$} & \multirow{2}{*}{$\mathrm{R}_{2}$} & \multirow[t]{2}{*}{$\mathrm{R}_{3}$} & \multirow{2}{*}{$\mathrm{R}_{4}$} & \multirow{2}{*}{$\begin{array}{c}\text { Yield } \\
(\%)\end{array}$} & \multirow{2}{*}{$\begin{array}{l}\text { M.p. } \\
\left({ }^{\circ} \mathrm{C}\right)\end{array}$} & \multirow{2}{*}{ Mol. formula $\left(M_{r}\right)$} & \multicolumn{3}{|c|}{$\begin{array}{c}\text { Elemental analysis (\%) } \\
\text { Calcd./found }\end{array}$} \\
\hline & & & & & & & & $\mathrm{C}$ & $\mathrm{H}$ & $\mathrm{N}$ \\
\hline $3 a$ & $\mathrm{OH}$ & $\mathrm{H}$ & $\mathrm{H}$ & $\mathrm{Cl}$ & 95 & $85-87$ & $\begin{array}{c}\mathrm{C}_{16} \mathrm{H}_{16} \mathrm{Cl}_{2} \mathrm{~N}_{2} \mathrm{O}_{2} \\
(339.22)\end{array}$ & $\begin{array}{l}56.65 \\
56.74\end{array}$ & $\begin{array}{l}4.73 \\
4.70\end{array}$ & $\begin{array}{l}8.28 \\
8.26\end{array}$ \\
\hline $3 b$ & $\mathrm{H}$ & $\mathrm{H}$ & $\mathrm{Cl}$ & $\mathrm{H}$ & 94 & $115-117$ & $\begin{array}{c}\mathrm{C}_{16} \mathrm{H}_{16} \mathrm{Cl}_{2} \mathrm{~N}_{2} \mathrm{O} \\
(323.22)\end{array}$ & $\begin{array}{l}59.46 \\
59.58\end{array}$ & $\begin{array}{l}4.96 \\
4.89\end{array}$ & $\begin{array}{l}8.69 \\
8.65\end{array}$ \\
\hline $3 c$ & $\mathrm{OH}$ & I & $\mathrm{H}$ & $\mathrm{Cl}$ & 93 & $141-143$ & $\begin{array}{c}\mathrm{C}_{16} \mathrm{H}_{15} \mathrm{Cl}_{2} \mathrm{IN}_{2} \mathrm{O}_{2} \\
(465.12)\end{array}$ & $\begin{array}{l}41.37 \\
41.35\end{array}$ & $\begin{array}{l}3.23 \\
3.26\end{array}$ & $\begin{array}{l}6.03 \\
6.08\end{array}$ \\
\hline $3 d$ & $\mathrm{OH}$ & $\mathrm{Br}$ & $\mathrm{H}$ & $\mathrm{Cl}$ & 94 & $132-134$ & $\begin{array}{c}\mathrm{C}_{16} \mathrm{H}_{15} \mathrm{BrCl}_{2} \mathrm{~N}_{2} \mathrm{O}_{2} \\
(418.12)\end{array}$ & $\begin{array}{l}46.04 \\
46.01\end{array}$ & $\begin{array}{l}3.59 \\
3.51\end{array}$ & $\begin{array}{l}6.74 \\
6.82\end{array}$ \\
\hline $3 e$ & $\mathrm{OH}$ & $\mathrm{H}$ & $\mathrm{CH}_{3}$ & $\mathrm{Cl}$ & 92 & $105-107$ & $\begin{array}{c}\mathrm{C}_{17} \mathrm{H}_{18} \mathrm{Cl}_{2} \mathrm{~N}_{2} \mathrm{O}_{2} \\
(353.25)\end{array}$ & $\begin{array}{l}57.81 \\
57.88\end{array}$ & $\begin{array}{l}5.14 \\
5.11\end{array}$ & $\begin{array}{l}7.95 \\
7.86\end{array}$ \\
\hline $3 f$ & $\mathrm{OH}$ & I & $\mathrm{CH}_{3}$ & $\mathrm{Cl}$ & 88 & $126-128$ & $\begin{array}{c}\mathrm{C}_{17} \mathrm{H}_{17} \mathrm{Cl}_{2} \mathrm{IN}_{2} \mathrm{O}_{2} \\
(479.15)\end{array}$ & $\begin{array}{l}42.67 \\
42.62\end{array}$ & $\begin{array}{l}3.58 \\
3.46\end{array}$ & $\begin{array}{l}5.85 \\
5.87\end{array}$ \\
\hline $3 g$ & $\mathrm{OH}$ & $\mathrm{Br}$ & $\mathrm{CH}_{3}$ & $\mathrm{Cl}$ & 91 & $98-100$ & $\begin{array}{c}\mathrm{C}_{17} \mathrm{H}_{17} \mathrm{BrCl}_{2} \mathrm{~N}_{2} \mathrm{O}_{2} \\
(432.15)\end{array}$ & $\begin{array}{l}47.26 \\
47.34\end{array}$ & $\begin{array}{l}3.97 \\
3.85\end{array}$ & $\begin{array}{l}6.48 \\
6.52\end{array}$ \\
\hline $3 \mathrm{~h}$ & $\mathrm{H}$ & $\mathrm{H}$ & $\mathrm{NH}_{2}$ & $\mathrm{H}$ & 89 & 110-112 & $\begin{array}{c}\mathrm{C}_{16} \mathrm{H}_{18} \mathrm{ClN}_{3} \mathrm{O} \\
(303.79)\end{array}$ & $\begin{array}{l}63.26 \\
63.19\end{array}$ & $\begin{array}{l}5.93 \\
5.95\end{array}$ & $\begin{array}{l}13.80 \\
13.84\end{array}$ \\
\hline $3 \mathbf{i}$ & $\mathrm{H}$ & $\mathrm{H}$ & $\mathrm{OCH}_{3}$ & $\mathrm{H}$ & 89 & $120-122$ & $\begin{array}{c}\mathrm{C}_{17} \mathrm{H}_{19} \mathrm{ClN}_{2} \mathrm{O}_{2} \\
(318.81)\end{array}$ & $\begin{array}{l}64.05 \\
64.11\end{array}$ & $\begin{array}{l}5.97 \\
5.91\end{array}$ & $\begin{array}{l}8.80 \\
8.85\end{array}$ \\
\hline $3 \mathbf{j}$ & $\mathrm{OH}$ & I & $\mathrm{H}$ & $\mathrm{CH}_{3}$ & 90 & $92-94$ & $\begin{array}{c}\mathrm{C}_{17} \mathrm{H}_{18} \mathrm{ClIN}_{2} \mathrm{O}_{2} \\
(444.70)\end{array}$ & $\begin{array}{l}45.92 \\
45.86\end{array}$ & $\begin{array}{l}4.08 \\
4.12\end{array}$ & $\begin{array}{l}6.32 \\
6.38\end{array}$ \\
\hline $3 k$ & $\mathrm{OH}$ & $\mathrm{H}$ & $\mathrm{H}$ & $\mathrm{Cl}$ & 91 & $198-200$ & $\begin{array}{c}\mathrm{C}_{18} \mathrm{H}_{12} \mathrm{Cl}_{2} \mathrm{~N}_{2} \mathrm{O}_{2} \mathrm{~S} \\
(391.28)\end{array}$ & $\begin{array}{l}55.24 \\
55.21\end{array}$ & $\begin{array}{l}3.06 \\
3.09\end{array}$ & $\begin{array}{l}7.16 \\
7.11\end{array}$ \\
\hline 31 & $\mathrm{H}$ & $\mathrm{H}$ & $\mathrm{Cl}$ & $\mathrm{H}$ & 92 & $156-158$ & $\begin{array}{c}\mathrm{C}_{18} \mathrm{H}_{12} \mathrm{Cl}_{2} \mathrm{~N}_{2} \mathrm{OS} \\
(375.28)\end{array}$ & $\begin{array}{l}57.60 \\
57.68\end{array}$ & $\begin{array}{l}3.20 \\
3.12\end{array}$ & $\begin{array}{l}7.46 \\
7.41\end{array}$ \\
\hline $3 m$ & $\mathrm{OH}$ & I & $\mathrm{H}$ & $\mathrm{Cl}$ & 92 & $142-144$ & $\begin{array}{c}\mathrm{C}_{18} \mathrm{H}_{11} \mathrm{Cl}_{2} \mathrm{IN}_{2} \mathrm{O}_{2} \mathrm{~S} \\
(517.17)\end{array}$ & $\begin{array}{l}41.77 \\
41.72\end{array}$ & $\begin{array}{l}2.12 \\
2.10\end{array}$ & $\begin{array}{l}5.41 \\
5.48\end{array}$ \\
\hline $3 n$ & $\mathrm{OH}$ & $\mathrm{Br}$ & $\mathrm{H}$ & $\mathrm{Cl}$ & 93 & $161-163$ & $\begin{array}{c}\mathrm{C}_{18} \mathrm{H}_{11} \mathrm{BrCl}_{2} \mathrm{~N}_{2} \mathrm{O}_{2} \mathrm{~S} \\
(470.17)\end{array}$ & $\begin{array}{l}45.98 \\
45.91\end{array}$ & $\begin{array}{l}2.36 \\
2.31\end{array}$ & $\begin{array}{l}5.98 \\
5.85\end{array}$ \\
\hline 30 & $\mathrm{OH}$ & $\mathrm{H}$ & $\mathrm{CH}_{3}$ & $\mathrm{Cl}$ & 89 & $128-130$ & $\begin{array}{c}\mathrm{C}_{19} \mathrm{H}_{14} \mathrm{Cl}_{2} \mathrm{~N}_{2} \mathrm{O}_{2} \mathrm{~S} \\
(405.31)\end{array}$ & $\begin{array}{l}56.29 \\
56.21\end{array}$ & $\begin{array}{l}3.45 \\
3.48\end{array}$ & $\begin{array}{l}6.91 \\
6.95\end{array}$ \\
\hline $3 p$ & $\mathrm{OH}$ & I & $\mathrm{CH}_{3}$ & $\mathrm{Cl}$ & 88 & $134-136$ & $\begin{array}{c}\mathrm{C}_{19} \mathrm{H}_{13} \mathrm{Cl}_{2} \mathrm{IN}_{2} \mathrm{O}_{2} \mathrm{~S} \\
(531.20)\end{array}$ & $\begin{array}{l}42.96 \\
42.89\end{array}$ & $\begin{array}{l}2.46 \\
2.52\end{array}$ & $\begin{array}{l}5.28 \\
5.35\end{array}$ \\
\hline
\end{tabular}

cillium chrysogenum and Fusarium moneliforme. The results were compared with penicillin and nystatin. All the culture strains of fungi were maintained on a potato dextrose agar (PDA) slant at $27 \pm 2{ }^{\circ} \mathrm{C}$ for $24-28 \mathrm{~h}$, until sporulation. Spores were transferred into $5 \mathrm{~mL}$ of sterile distilled water containing $1 \%$ Tween- 80 (to suspend the spores properly). The spores were counted with a haemocytometer $\left(10^{6} \mathrm{CFU} \mathrm{mL} \mathrm{mL}^{-1}\right)$. Sterile PDA plates containing $2 \%$ agar were prepared; $0.1 \mathrm{~mL}$ of each fungal spore suspension was spread on 
B. S. Dawane et al.: An improved procedure for synthesis of some new 1,3-diaryl-2-propen-1-ones using PEG-400 as a recyclable solvent and their antimicrobial evaluation, Acta Pharm. 59 (2009) 473-482.

Table II. Spectral data of newly synthesized compounds

\begin{tabular}{|c|c|c|c|c|}
\hline $\begin{array}{l}\text { Compd. } \\
\text { No. }\end{array}$ & $\begin{array}{c}\text { Mass } \\
(m / z, \%)\end{array}$ & $\begin{array}{c}\mathrm{IR} \\
\left(\mathrm{cm}^{-1}\right)\end{array}$ & $\begin{array}{l}{ }^{1} \mathrm{H} \text { NMR } \\
(\delta, \mathrm{ppm})\end{array}$ & $\begin{array}{c}{ }^{13} \mathrm{C} \text { NMR } \\
(\delta, \mathrm{ppm})\end{array}$ \\
\hline $3 a$ & $\begin{array}{l}343[\mathrm{M}+4](8), \\
341[\mathrm{M}+2](18), \\
339\left[\mathrm{M}^{+}\right](25)\end{array}$ & $\begin{array}{l}3338(-\mathrm{NH}), \\
3159(-\mathrm{OH}) \\
1651(>\mathrm{C}=\mathrm{O}), \\
1599(-\mathrm{C}=\mathrm{N})\end{array}$ & $\begin{array}{l}0.93\left(\mathrm{t}, 3 \mathrm{H},-\mathrm{CH}_{3}\right), 1.31(\mathrm{~m}, 2 \mathrm{H}, \\
\left.\mathrm{CH}_{2}\right), 1.65\left(\mathrm{~m}, 2 \mathrm{H},-\mathrm{CH}_{2}-\right), 2.76 \\
\left(\mathrm{t}, 2 \mathrm{H}, \mathrm{CH}_{2}\right), 7.02-7.96(\mathrm{~m}, 5 \mathrm{H}, \\
\mathrm{Ar}-\mathrm{H}+\mathrm{CH}=\mathrm{CH}), 8.15(\mathrm{~s}, 1 \mathrm{H}, \\
-\mathrm{NH}), 11.61(\mathrm{~s}, 1 \mathrm{H},-\mathrm{OH})\end{array}$ & - \\
\hline $3 b$ & $\begin{array}{l}327[\mathrm{M}+4](4), \\
325[\mathrm{M}+2](14), \\
323\left[\mathrm{M}^{+}\right](22)\end{array}$ & $\begin{array}{l}3335(-\mathrm{NH}), \\
3142(-\mathrm{OH}), \\
1651(>\mathrm{C}=\mathrm{O}) \\
1598(-\mathrm{C}=\mathrm{N})\end{array}$ & $\begin{array}{l}0.93\left(\mathrm{t}, 3 \mathrm{H},-\mathrm{CH}_{3}\right), 1.32(\mathrm{~m}, 2 \mathrm{H}, \\
\left.\mathrm{CH}_{2}\right), 1.64\left(\mathrm{~m}, 2 \mathrm{H},-\mathrm{CH}_{2^{-}}\right), 2.72 \\
\left(\mathrm{t}, 2 \mathrm{H}, \mathrm{CH}_{2}\right), 7.12-7.98(\mathrm{~m}, 6 \mathrm{H}, \\
\mathrm{Ar}-\mathrm{H}+\mathrm{CH}=\mathrm{CH}), 8.16(\mathrm{~s}, 1 \mathrm{H}, \\
-\mathrm{NH})\end{array}$ & $\begin{array}{l}14\left(\mathrm{CH}_{3}\right), 22\left(\mathrm{CH}_{2}\right), \\
29\left(\mathrm{CH}_{2}\right), 30,\left(\mathrm{CH}_{2}\right), \\
117,122,128,129(2 \\
\times \mathrm{C}), 130(2 \times \mathrm{C}) \\
134,136,138,152, \\
187(\mathrm{C}=\mathrm{O})\end{array}$ \\
\hline $3 c$ & $\begin{array}{l}469[\mathrm{M}+4](5), \\
467[\mathrm{M}+2](19), \\
465\left[\mathrm{M}^{+}\right](28)\end{array}$ & $\begin{array}{l}3332(-\mathrm{NH}), \\
3156(-\mathrm{OH}) \\
1654(>\mathrm{C}=\mathrm{O}), \\
1599(-\mathrm{C}=\mathrm{N})\end{array}$ & $\begin{array}{l}0.93\left(\mathrm{t}, 3 \mathrm{H},-\mathrm{CH}_{3}\right), 1.32(\mathrm{~m}, 2 \mathrm{H}, \\
\left.-\mathrm{CH}_{2}\right), 1.65\left(\mathrm{~m}, 2 \mathrm{H},-\mathrm{CH}_{2^{-}}\right) \\
2.74\left(\mathrm{t}, 2 \mathrm{H}, \mathrm{CH}_{2}\right), 7.05-7.99(\mathrm{~m}, \\
4 \mathrm{H}, \mathrm{Ar}-\mathrm{H}+\mathrm{CH}=\mathrm{CH}), 8.18(\mathrm{~s}, \\
1 \mathrm{H},-\mathrm{NH}), 11.45(\mathrm{~s}, 1 \mathrm{H},-\mathrm{OH})\end{array}$ & $\begin{array}{l}14\left(\mathrm{CH}_{3}\right), 22\left(\mathrm{CH}_{2}\right), \\
28\left(\mathrm{CH}_{2}\right), 30\left(\mathrm{CH}_{2}\right), \\
116,122,123,128, \\
129,131,132,135, \\
138,139,153,189 \\
(\mathrm{C}=\mathrm{O})\end{array}$ \\
\hline $3 d$ & $\begin{array}{l}424[\mathrm{M}+6](6), \\
422[\mathrm{M}+4](25), \\
420[\mathrm{M}+2](55), \\
418\left[\mathrm{M}^{+}\right](34)\end{array}$ & $\begin{array}{l}3331(-\mathrm{NH}), \\
3148(-\mathrm{OH}), \\
1652(>\mathrm{C}=\mathrm{O}), \\
1595(-\mathrm{C}=\mathrm{N})\end{array}$ & $\begin{array}{l}0.90\left(\mathrm{t}, 3 \mathrm{H},-\mathrm{CH}_{3}\right), 1.33(\mathrm{~m}, 2 \mathrm{H}, \\
\left.-\mathrm{CH}_{2}\right), 1.66\left(\mathrm{~m}_{2}, 2 \mathrm{H},-\mathrm{CH}_{2}-\right) \\
2.81\left(\mathrm{t}, 2 \mathrm{H}, \mathrm{CH}_{2}\right), 7.26-8.05(\mathrm{~m}, \\
4 \mathrm{H}, \mathrm{Ar}-\mathrm{H}+\mathrm{CH}=\mathrm{CH}), 8.12(\mathrm{~s}, \\
1 \mathrm{H},-\mathrm{NH}), 11.58(\mathrm{~s}, 1 \mathrm{H},-\mathrm{OH})\end{array}$ & - \\
\hline $3 e$ & $\begin{array}{l}357[\mathrm{M}+4](5), \\
355[\mathrm{M}+2](17), \\
353\left[\mathrm{M}^{+}\right](26)\end{array}$ & $\begin{array}{l}3331(-\mathrm{NH}), \\
3146(-\mathrm{OH}), \\
1649(>\mathrm{C}=\mathrm{O}), \\
1597(-\mathrm{C}=\mathrm{N})\end{array}$ & $\begin{array}{l}0.93\left(\mathrm{t}, 3 \mathrm{H},-\mathrm{CH}_{3}\right), 1.33(\mathrm{~m}, 2 \mathrm{H}, \\
\left.-\mathrm{CH}_{2}\right), 1.65\left(\mathrm{~m}, 2 \mathrm{H},-\mathrm{CH}_{2}-\right), \\
2.33\left(\mathrm{~s}, 3 \mathrm{H}, \mathrm{CH}_{3}\right), 2.71(\mathrm{t}, 2 \mathrm{H},- \\
\left.\mathrm{CH}_{2}\right), 7.05-7.94(\mathrm{~m}, 4 \mathrm{H}, \mathrm{Ar}-\mathrm{H}+ \\
\mathrm{CH}=\mathrm{CH}), 8.16(\mathrm{~s}, 1 \mathrm{H},-\mathrm{NH}), \\
11.56(\mathrm{~s}, 1 \mathrm{H},-\mathrm{OH})\end{array}$ & - \\
\hline $3 f$ & $\begin{array}{l}483[\mathrm{M}+4](4), \\
481[\mathrm{M}+2](12), \\
479\left[\mathrm{M}^{+}\right](18)\end{array}$ & $\begin{array}{l}3335(-\mathrm{NH}), \\
3151(-\mathrm{OH}), \\
1650(>\mathrm{C}=\mathrm{O}), \\
1598(-\mathrm{C}=\mathrm{N})\end{array}$ & $\begin{array}{l}0.95\left(\mathrm{t}, 3 \mathrm{H},-\mathrm{CH}_{3}\right), 1.36(\mathrm{~m}, 2 \mathrm{H}, \\
\left.-\mathrm{CH}_{2}\right), 1.62\left(\mathrm{~m}, 2 \mathrm{H},-\mathrm{CH}_{2}-\right), \\
2.38\left(\mathrm{~s}, 3 \mathrm{H}, \mathrm{CH}_{3}\right), 2.76(\mathrm{t}, 2 \mathrm{H},- \\
\left.\mathrm{CH}_{2}\right), 7.08-7.99(\mathrm{~m}, 3 \mathrm{H}, \mathrm{Ar}-\mathrm{H}+ \\
\mathrm{CH}=\mathrm{CH}), 8.20(\mathrm{~s}, 1 \mathrm{H},-\mathrm{NH}), \\
11.76(\mathrm{~s}, 1 \mathrm{H},-\mathrm{OH})\end{array}$ & - \\
\hline $3 g$ & $\begin{array}{l}438[\mathrm{M}+6](8), \\
436[\mathrm{M}+4](33), \\
434[\mathrm{M}+2](69), \\
432\left[\mathrm{M}^{+}\right](42)\end{array}$ & $\begin{array}{l}3332(-\mathrm{NH}), \\
3162(-\mathrm{OH}), \\
1651(>\mathrm{C}=\mathrm{O}), \\
1599(-\mathrm{C}=\mathrm{N})\end{array}$ & $\begin{array}{l}0.93\left(\mathrm{t}, 3 \mathrm{H},-\mathrm{CH}_{3}\right), 1.31(\mathrm{~m}, 2 \mathrm{H}, \\
\left.-\mathrm{CH}_{2}\right), 1.67\left(\mathrm{~m}, 2 \mathrm{H},-\mathrm{CH}_{2}-\right) \\
2.35\left(\mathrm{~s}, 3 \mathrm{H}, \mathrm{CH}_{3}\right), 2.76(\mathrm{t}, 2 \mathrm{H},- \\
\left.\mathrm{CH}_{2}\right), 7.15-8.12(\mathrm{~m}, 3 \mathrm{H}, \mathrm{Ar}-\mathrm{H}+ \\
\mathrm{CH}=\mathrm{CH}), 8.21(\mathrm{~s}, 1 \mathrm{H},-\mathrm{NH}), \\
11.62(\mathrm{~s}, 1 \mathrm{H},-\mathrm{OH})\end{array}$ & - \\
\hline $3 h$ & $\begin{array}{l}305[\mathrm{M}+2](8) \\
303\left[\mathrm{M}^{+}\right](18)\end{array}$ & $\begin{array}{l}3398\left(-\mathrm{NH}_{2}\right), \\
1648(>\mathrm{C}=\mathrm{O}), \\
1596(-\mathrm{C}=\mathrm{N})\end{array}$ & $\begin{array}{l}0.93\left(\mathrm{t}, 3 \mathrm{H},-\mathrm{CH}_{3}\right), 1.32(\mathrm{~m}, 2 \mathrm{H}, \\
\left.-\mathrm{CH}_{2}\right), 1.65\left(\mathrm{~m}, 2 \mathrm{H},-\mathrm{CH}_{2}-\right) \\
2.74\left(\mathrm{t}, 2 \mathrm{H}, \mathrm{CH}_{2}\right), 4.82(\mathrm{bs}, 2 \mathrm{H}, \\
\left.\mathrm{NH}_{2}\right), 7.12-8.10(\mathrm{~m}, 6 \mathrm{H}, \mathrm{Ar}-\mathrm{H}+ \\
\mathrm{CH}=\mathrm{CH}), 8.21(\mathrm{~s}, 1 \mathrm{H},-\mathrm{NH})\end{array}$ & - \\
\hline
\end{tabular}


B. S. Dawane et al.: An improved procedure for synthesis of some new 1,3-diaryl-2-propen-1-ones using PEG-400 as a recyclable solvent and their antimicrobial evaluation, Acta Pharm. 59 (2009) 473-482.

\begin{tabular}{|c|c|c|c|c|}
\hline $3 i$ & $\begin{array}{l}320[\mathrm{M}+2](10), \\
318\left[\mathrm{M}^{+}\right](26)\end{array}$ & $\begin{array}{l}3326(-\mathrm{NH}) \\
1651(>\mathrm{C}=\mathrm{O}) \\
1598(-\mathrm{C}=\mathrm{N})\end{array}$ & $\begin{array}{l}0.93\left(\mathrm{t}, 3 \mathrm{H},-\mathrm{CH}_{3}\right), 1.35(\mathrm{~m}, 2 \mathrm{H}, \\
\left.-\mathrm{CH}_{2}\right), 1.65\left(\mathrm{~m}, 2 \mathrm{H},-\mathrm{CH}_{2}-\right), \\
2.76\left(\mathrm{t}, 2 \mathrm{H}, \mathrm{CH}_{2}\right), 3.38(\mathrm{~s}, 3 \mathrm{H}, \\
\left.\mathrm{OCH}_{3}\right), 7.05-8.06(\mathrm{~m}, 6 \mathrm{H}, \mathrm{Ar}-\mathrm{H} \\
+\mathrm{CH}=\mathrm{CH}), 8.20(\mathrm{~s}, 1 \mathrm{H},-\mathrm{NH})\end{array}$ & - \\
\hline $3 j$ & $\begin{array}{l}446[\mathrm{M}+2](6) \\
444\left[\mathrm{M}^{+}\right](15)\end{array}$ & $\begin{array}{l}3332(-\mathrm{NH}), \\
3163(-\mathrm{OH}), \\
1651(>\mathrm{C}=\mathrm{O}), \\
1589(-\mathrm{C}=\mathrm{N})\end{array}$ & $\begin{array}{l}0.94\left(\mathrm{t}, 3 \mathrm{H},-\mathrm{CH}_{3}\right), 1.34(\mathrm{~m}, 2 \mathrm{H}, \\
\left.-\mathrm{CH}_{2}\right), 1.63\left(\mathrm{~m}, 2 \mathrm{H},-\mathrm{CH}_{2}-\right) \\
2.76\left(\mathrm{t}, 2 \mathrm{H}, \mathrm{CH}_{2}\right), 2.38(\mathrm{~s}, 3 \mathrm{H}, \\
\left.\mathrm{CH}_{3}\right), 7.10-8.13(\mathrm{~m}, 4 \mathrm{H}, \mathrm{Ar}-\mathrm{H}+ \\
\mathrm{CH}=\mathrm{CH}), 8.25(\mathrm{~s}, 1 \mathrm{H},-\mathrm{NH}), \\
11.48(\mathrm{~s}, 1 \mathrm{H},-\mathrm{OH})\end{array}$ & - \\
\hline $3 k$ & $\begin{array}{l}395[\mathrm{M}+4](8), \\
393[\mathrm{M}+2](25), \\
391\left[\mathrm{M}^{+}\right](34)\end{array}$ & $\begin{array}{l}3299\left(-\mathrm{NH}_{2}\right), \\
3130(-\mathrm{OH}) \\
1630(>\mathrm{C}=\mathrm{O}), \\
1586(-\mathrm{C}=\mathrm{N})\end{array}$ & $\begin{array}{l}7.10-8.25(\mathrm{~m}, 9 \mathrm{H}, \mathrm{Ar}-\mathrm{H}+ \\
\mathrm{CH}=\mathrm{CH}), 8.33(\mathrm{~s}, 2 \mathrm{H},-\mathrm{NH} 2) \text {, } \\
11.84(\mathrm{~s}, 1 \mathrm{H},-\mathrm{OH})\end{array}$ & - \\
\hline 31 & $\begin{array}{l}379[\mathrm{M}+4](5), \\
377[\mathrm{M}+2](20), \\
375\left[\mathrm{M}^{+}\right](28)\end{array}$ & $\begin{array}{l}3308\left(-\mathrm{NH}_{2}\right) \\
1636(>\mathrm{C}=\mathrm{O}) \\
1592(-\mathrm{C}=\mathrm{N})\end{array}$ & $\begin{array}{l}7.02-8.18(\mathrm{~m}, 10 \mathrm{H}, \mathrm{Ar}-\mathrm{H}+ \\
\mathrm{CH}=\mathrm{CH}), 8.36\left(\mathrm{~s}, 2 \mathrm{H},-\mathrm{NH}_{2}\right)\end{array}$ & - \\
\hline $3 \mathrm{~m}$ & $\begin{array}{l}521[\mathrm{M}+4](6), \\
519[\mathrm{M}+2](18), \\
517\left[\mathrm{M}^{+}\right](25)\end{array}$ & $\begin{array}{l}3298\left(-\mathrm{NH}_{2}\right) \\
3162(-\mathrm{OH}) \\
1632(>\mathrm{C}=\mathrm{O}) \\
1591(-\mathrm{C}=\mathrm{N})\end{array}$ & $\begin{array}{l}7.08-8.22(\mathrm{~m}, 8 \mathrm{H}, \mathrm{Ar}-\mathrm{H}+ \\
\mathrm{CH}=\mathrm{CH}), 8.34\left(\mathrm{~s}, 2 \mathrm{H},-\mathrm{NH}_{2}\right) \\
11.51(\mathrm{~s}, 1 \mathrm{H},-\mathrm{OH})\end{array}$ & - \\
\hline $3 n$ & $\begin{array}{l}476[\mathrm{M}+6](8), \\
474[\mathrm{M}+4](35), \\
472[\mathrm{M}+2](76), \\
470\left[\mathrm{M}^{+}\right](46)\end{array}$ & $\begin{array}{l}3316\left(-\mathrm{NH}_{2}\right), \\
3146(-\mathrm{OH}) \\
1635(>\mathrm{C}=\mathrm{O}), \\
1598(-\mathrm{C}=\mathrm{N})\end{array}$ & $\begin{array}{l}7.05-8.25(\mathrm{~m}, 8 \mathrm{H}, \mathrm{Ar}-\mathrm{H}+ \\
\mathrm{CH}=\mathrm{CH}), 8.32\left(\mathrm{~s}, 2 \mathrm{H},-\mathrm{NH}_{2}\right) \\
11.78(\mathrm{~s}, 1 \mathrm{H},-\mathrm{OH})\end{array}$ & - \\
\hline 30 & $\begin{array}{l}409[\mathrm{M}+4](6), \\
407[\mathrm{M}+2](25), \\
405\left[\mathrm{M}^{+}\right](35)\end{array}$ & $\begin{array}{l}3289\left(-\mathrm{NH}_{2}\right) \\
3158(-\mathrm{OH}) \\
1635(>\mathrm{C}=\mathrm{O}) \\
1596(-\mathrm{C}=\mathrm{N})\end{array}$ & $\begin{array}{l}2.38\left(\mathrm{~s}, 3 \mathrm{H}, \mathrm{CH}_{3}\right), 7.06-8.20(\mathrm{~m}, \\
8 \mathrm{H}, \mathrm{Ar}-\mathrm{H}+\mathrm{CH}=\mathrm{CH}), 8.33(\mathrm{~s}, \\
\left.2 \mathrm{H},-\mathrm{NH}_{2}\right), 11.21(\mathrm{~s}, 1 \mathrm{H},-\mathrm{OH})\end{array}$ & - \\
\hline $3 p$ & $\begin{array}{l}535[\mathrm{M}+4](5), \\
533[\mathrm{M}+2](20), \\
531[\mathrm{M}+](28)\end{array}$ & $\begin{array}{l}3325\left(-\mathrm{NH}_{2}\right), \\
3172(-\mathrm{OH}) \\
1631(>\mathrm{C}=\mathrm{O}), \\
1595(-\mathrm{C}=\mathrm{N})\end{array}$ & $\begin{array}{l}2.42\left(\mathrm{~s}, 3 \mathrm{H}, \mathrm{CH}_{3}\right), 7.08-8.26(\mathrm{~m}, \\
7 \mathrm{H}, \mathrm{Ar}-\mathrm{H}+\mathrm{CH}=\mathrm{CH}), 8.32(\mathrm{~s} \\
\left.2 \mathrm{H},-\mathrm{NH}_{2}\right), 1.182(\mathrm{~s}, 1 \mathrm{H},-\mathrm{OH})\end{array}$ & - \\
\hline
\end{tabular}

each plate and incubated at $27 \pm 2{ }^{\circ} \mathrm{C}$ for $12 \mathrm{~h}$. After incubation, a hole was made using a sterile cork borer and each agar well was filled with $0.1 \mathrm{~mL}$ chalcone solution of 50,100 and $250 \mu \mathrm{g} \mathrm{mL}^{-1}$ separately to get the minimum inhibitory concentration (MIC) value of chalcones. Dimethyl sulphoxide (DMSO) was used as a solvent for chalcones and as well as a control, while distilled water used as solvent for standard drugs.

The plates were kept in refrigerator for 20 minutes for diffusion and then incubated at $27 \pm 2{ }^{\circ} \mathrm{C}$ for $24-28 \mathrm{~h}$ in an incubator. After incubation, the zone of inhibition of compounds was measured in $\mathrm{mm}$ and standard and minimum inhibitory concentrations (MICs) were noted. The results of antimicrobial studies are given in Table III. 
B. S. Dawane et al:: An improved procedure for synthesis of some new 1,3-diaryl-2-propen-1-ones using PEG-400 as a recyclable solvent and their antimicrobial evaluation, Acta Pharm. 59 (2009) 473-482.

Table III. Antimicrobial activity of synthesized compounds ${ }^{a}$

\begin{tabular}{ccccccccc}
\hline & \multicolumn{7}{c}{ Zone of inhibition, mm (MIC value, $\left.\mu \mathrm{g} \mathrm{L} \mathrm{L}^{-1}\right)$} \\
$\begin{array}{c}\text { Compd. } \\
\text { No. }\end{array}$ & \multicolumn{7}{c}{ Bacteria } & \multicolumn{5}{c}{ Fungi } \\
\cline { 2 - 9 } & Ec & St & Sa & Bs & An & Af & Pc & Fm \\
\hline 3a & $13(100)$ & - & $15(100)$ & $12(100)$ & $12(100)$ & $14(100)$ & $12(100)$ & $15(100)$ \\
3b & $12(100)$ & $13(100)$ & $13(100)$ & $16(100)$ & $15(100)$ & - & $14(100)$ & - \\
3c & $15(50)$ & $18(50)$ & $16(50)$ & $20(50)$ & $18(50)$ & $20(50)$ & $19(50)$ & $21(50)$ \\
3d & $10(100)$ & $12(100)$ & - & $9(100)$ & $16(50)$ & $19(50)$ & $18(50)$ & $16(50)$ \\
3e & $18(50)$ & $16(50)$ & $19(50)$ & $20(50)$ & $18(50)$ & $20(50)$ & $19(50)$ & $18(50)$ \\
3f & $11(100)$ & $12(100)$ & $14(100)$ & $11(100)$ & $14(100)$ & $15(100)$ & $16(100)$ & $12(100)$ \\
3g & $16(50)$ & $20(50)$ & $18(50)$ & $14(50)$ & $19(50)$ & $20(50)$ & $22(50)$ & $20(50)$ \\
3h & $12(100)$ & - & $13(100)$ & $10(100)$ & $16(100)$ & - & $14(100)$ & $18(100)$ \\
3i & $10(100)$ & $12(100)$ & $9(100)$ & $13(100)$ & $15(100)$ & - & $16(100)$ & $15(100)$ \\
3j & $17(50)$ & $19(50)$ & $18(50)$ & $16(50)$ & $19(50)$ & $20(50)$ & $19(50)$ & $22(50)$ \\
3k & - & $13(100)$ & $12(100)$ & $14(100)$ & $16(100)$ & $15(100)$ & $16(100)$ & $14(100)$ \\
31 & $15(100)$ & $12(100)$ & - & $11(100)$ & $19(100)$ & $18(100)$ & $19(100)$ & $18(100)$ \\
3m & $14(100)$ & $11(100)$ & $13(100)$ & $8(100)$ & $18(100)$ & $16(100)$ & - & $19(50)$ \\
3n & $19(50)$ & $14(50)$ & $16(50)$ & $15(50)$ & $20(50)$ & $19(50)$ & $20(50)$ & $21(50)$ \\
30 & $13(100)$ & $11(100)$ & $12(100)$ & $6(100)$ & $16(100)$ & $18(100)$ & $15(100)$ & $16(100)$ \\
3p & $16(100)$ & $12(100)$ & $14(100)$ & $10(100)$ & $15(100)$ & $14(100)$ & $19(100)$ & $18(100)$ \\
Penicillin & $22(50)$ & $22(50)$ & $24(50)$ & $24(50)$ & NA & NA & NA & NA \\
Nystatin & NA & NA & NA & NA & $20(50)$ & $22(50)$ & $24(50)$ & $24(50)$ \\
\hline & & & & & & & &
\end{tabular}

a Solvents: DMSO, water

Ec - Escherichia coli, St - Salmonella typhi, Sa - Staphylococcus aureus, Bs - Bacillus subtillis, An - Aspergillus niger, Af-Aspergillus flavus, Fm - Fusarium moneliforme, Pc - Penicillium chrysogenum, (-) - MIC > $100 \mu \mathrm{g} \mathrm{L} \mathrm{L}^{-1}$, NA - not applicable

\section{RESULTS AND DISCUSSION}

The Claisen-Schmidt condensation (15) is an important C-C bond formation for the synthesis of 1,3-diaryl-2-propen-1-ones (chalcones). It is generally carried out by the use of strong bases such as $\mathrm{NaOH}$ or $\mathrm{KOH}$ in polar solvents (MeOH or DMF). The aim of the present study was to develop an efficient protocol using PEG-400 as a recyclable reaction solvent to obtain 1,3-diaryl-2-propen-1-ones with good to excellent yields in a short span of time without formation of any side product. 
B. S. Dawane et al.: An improved procedure for synthesis of some new 1,3-diaryl-2-propen-1-ones using PEG-400 as a recyclable solvent and their antimicrobial evaluation, Acta Pharm. 59 (2009) 473-482.<smiles>[R]c1cc(C(C)=O)c([R1])c([R2])c1[R3]</smiles>

1<smiles>[Z11]/C=C/C(=O)c1cc([R4])c([R])c([R])c1[R1]</smiles>

3

2<smiles>CCCCc1nc(Cl)c(C=O)[nH]1</smiles>

$3 a-j$<smiles>Nc1nc(-c2ccccc2)c(C=O)s1</smiles>
$3 k-p$

Scheme 1

First, we attempted condensation of 2-hydroxy-5-chloro-acetophenone with 2-butyl-4-chloro-5-formyl-imidazole using PEG-400 as a reaction solvent under alkaline conditions. The reaction was completed within $1 \mathrm{~h}$ and the corresponding product was obtained in $95 \%$ yield. Encouraged by the results, we turned our attention to a variety of acetophenones and hetero aldehydes. In all cases, the reaction proceeded efficiently in high yields at $40{ }^{\circ} \mathrm{C}$ using PEG-400 as an alternative reaction solvent.

IR spectra of chalcones showed characteristic bands at $1640-1650 \mathrm{~cm}^{-1}$ due to $>C=O$ stretching vibration. Lowering of normal $>\mathrm{C}=\mathrm{O}$ frequency was observed due to the presence of $-\mathrm{C}=\mathrm{C}$ stretching in chalcones. ${ }^{1} \mathrm{H}$ NMR spectra of the compounds showed characteristic doublet signals at $\delta 7.3$ and $7.8 \mathrm{ppm}$ due to alkene $\alpha, \beta$-protons, respectively. However, these doublets coalesced with aromatic protons. The phenolic proton $(2$ '-OH $)$ was observed as a singlet at $\delta 11-12.0 \mathrm{ppm}$ due to hydrogen bonding with the adjacent carbonyl group, while other aromatic and aliphatic protons were found at expected regions. ${ }^{13} \mathrm{C}$ NMR data of compounds exhibited chemical shift values of carbon atoms at $\delta$ $122\left(\mathrm{C}_{\alpha}\right), \delta 152-153\left(\mathrm{C}_{\beta}\right)$ and $\delta 187-189(>\mathrm{C}=\mathrm{O}) \mathrm{ppm}$ of the chalcones. The chemical shift values at $\delta 14,22,29$ and $30 \mathrm{ppm}$ corroborate the presence of the $n$-butyl group in the products. The mass spectra of compounds 3a-p showed molecular ion peaks corresponding to their molecular formula. Besides the molecular ion peak $\left[\mathrm{M}^{+}\right]$, the compounds showed $[\mathrm{M}+1]^{+}$(isotopic abundances), which confirmed the presence of halogen groups in respective compounds. The base peak was seen at $m / z 43$, corresponding to the $\mathrm{CH}_{3} \mathrm{C}=\mathrm{O}$ moiety.

All the synthesized compounds were tested for their in vitro antimicrobial activity. The results are given in Table III. Compounds $\mathbf{3 c}, \mathbf{3 e}, \mathbf{3 g}, \mathbf{3} \mathbf{j}$ and $\mathbf{3 n}$ showed good activity against all tested bacteria at concentration of $50 \mu \mathrm{g} \mathrm{mL}-1$. Compounds $3 \mathrm{c}$ and $3 \mathrm{e}$ showed a maximum zone of inhibition $(20 \mathrm{~mm})$ against $B$. subtillis compared to penicillin. Compounds $3 \mathbf{g}$ and $3 \mathbf{j}$ showed an effective zone of inhibition (19-20 $\mathrm{mm}$ ) against S. aureus in comparison with the standard. Compounds $3 \mathbf{a}, 3 \mathrm{~d}, 3 \mathbf{k}, 3 \mathbf{1}, 3 \mathrm{~m}$ and $3 \mathbf{3}$ were found to be less active against the tested bacterial strains $\left(M I C=100 \mu \mathrm{g} \mathrm{mL}^{-1}\right)$. Antifungal screening data showed that most of the compounds were active against all fungi. Compounds $3 \mathbf{c}$, 
B. S. Dawane et al.: An improved procedure for synthesis of some new 1,3-diaryl-2-propen-1-ones using PEG-400 as a recyclable solvent and their antimicrobial evaluation, Acta Pharm. 59 (2009) 473-482.

3d, 3e, 3g, 3j and $\mathbf{3 n}$ showed effective activity against all fungal strains at MIC of $50 \mu \mathrm{g}$ $\mathrm{mL}^{-1}$. Compound $3 \mathrm{n}$ showed comparabile activity against Aspergillus niger to standard drug, but compounds $\mathbf{3 g}, \mathbf{3} \mathbf{j}$ and $\mathbf{3 n}$ displayed an even stronger zone of inhibition (19-22 $\mathrm{mm})$ against all the tested fungi. On the other hand, compounds $3 \mathbf{b}, 3 \mathbf{h}$ and $3 \mathrm{~m}$ were found to be inactive against Aspergillus flavus, and compounds 3k, 31, 3o and $3 \mathbf{p}$ exhibited activities comparable to that of nystatin.

When structure and activity relationships are investigated, we can infer from the results that halogen at the $R_{2}$ position might be responsible for antibacterial and antifungal activity. Furthermore, it can be concluded that in compounds $\mathbf{3 g}$ and $\mathbf{3 j}$ bearing methyl group at $R_{3}$ or $R_{4}$ position in combination with $B r$ and $I$ at $R_{2}$, both groups enhanced activity. Further studies of the inhibitory activity of new compounds bearing methyl and halo substituents at different positions against important human pathogenic fungi are under way.

\section{CONCLUSIONS}

In conclusion, our protocol is a practical approach which uses PEG as a commercially available, low-cost, recyclable non-ionic solvent. In most cases, the reaction proceeded smoothly to produce the corresponding 1,3-diaryl-2-propen-1-ones. The reaction was clean and the products were obtained in excellent yields without formation of any side products. The substituted chalcone derivatives, 1-(5-chloro-2-hydroxy-3-iodo phenyl), 3-(2-butyl-5-chloro-3H-imidazol-4yl)-2-propen-1-one (3c), 1-(5-chloro-2-hydroxy-4-methyl phenyl), 3-(2-butyl-5-chloro-3H-imidazol-4yl)-2-propen-1-one (3e), 1-(3-bromo-5-chloro-2-hydroxy-4-methyl phenyl), 3-(2-butyl-5-chloro-3H-imidazol-4yl)-2-propen-1-one (3g), 1-(2-hydroxy-3-iodo-5-methyl phenyl), 3-(2-amino-4-(4-chlorophenyl)-thiazol-5-yl)-2-propen-1-one (3j), 1-(3-bromo-5-chloro-2-hydroxy phenyl), 3-(2-amino-4-(4-chlorophenyl)-thiazol-5-yl)-2-propen-1-one (3n) revealed significant antibacterial and antifungal activity. Compound 3n showed activity equivalent to that of the standard against Aspergillus niger. Considering the results obtained from antibacterial and antifungal tests together, it is noteworthy that the tested compounds were found more active towards fungi than bacteria.

Acknowledgements. - The authors are grateful to the Principal, Yeshwant Mahavidyalaya, Nanded, for providing laboratory facilities. Also, the authors thank the Director, IICT, Hyderabad, for providing necessary instrumental facilities.

\section{REFERENCES}

1. J. R. Dimmock, D. W. Elias, M. A. Beazely and N. M. Kandepu, Bioactivities of chalcones, Curr. Med. Chem. 6 (1999) 1125-1150.

2. M. L. Go, X. Wu and L. X. Liu, Chalcones: An update on cytotoxic and chemoprotective properties, Curr. Med. Chem. 12 (2005) 483-499.

3. Z. Nowakowka, A review of anti-infective and anti-inflammatory chalcones, Eur. J. Med. Chem. 42 (2007) 125-137; DOI: 10.1016/j.ejmech.2006.09.019. 
B. S. Dawane et al.: An improved procedure for synthesis of some new 1,3-diaryl-2-propen-1-ones using PEG-400 as a recyclable solvent and their antimicrobial evaluation, Acta Pharm. 59 (2009) 473-482.

4. C. N. Khobragade, R. G. Bodade, M. S. Shine, R. R. Deepa, R. B. Bhosale and B. S. Dawane, Microbial and xanthine dehydrogenase inhibitory activity of some flavones, J. Enzym Inhib. Med. Chem. 3 (2008) 341-346; DOI: 10.1080/14756360701608585.

5. S. Sogawa, Y. Nihro, H. Ueda, T. Miki, H. Matsumoto and T. Satoh, Protective effects of hydroxychalcones of free radical-induced cell damage, Biol. Pharm. Bull. 17 (1994) 251-256.

6. O. Nerya, R. Musa, S. Khatib, S. Tamir and J. Vaya, Chalcones as potent tyrosinase inhibitors: the effect of hydroxyl positions and numbers, Phytochemistry 65 (2004) 1389-1395; DOI: 10.1016/ j.phytochem.2004.04.016.

7. O. Prakash, A. Kumar, A. Sadana, R. Prakash, P. S. Singh, M. R. Claramunt, D. Sanz, I. Alkortac and J. Elguero, Study of the reaction of chalcone analogs of dehydroacetic acid and o-aminothiophenol: synthesis and structure of 1,5-benzothiazepines and 1,4-benzothiazines, Tetrahedron 61 (2005) 6642-6651; DOI: 10.1016/j.tet.2005.03.035.

8. R. Y. Prasad, L. A. Rao, L. Prasoona, K. Murali and R. P. Kumar, Synthesis and antidepressant activity of some 1,3,5-triphenyl-2-pyrazolines and 3-(2"-hydroxy naphthalen-1"-yl)-1,5-diphenyl-2-pyrazolines, Bioorg. Med. Chem. Lett. 15 (2005) 5030-5034; DOI: 10.1016/j.bmcl.2005.08.040.

9. S. Raghavan and K. Anuradha, Solid-phase synthesis of 1,4-diketones by thiazolium salt promoted addition of aldehydes to chalcones, Tetrahedron Lett. 43 (2002) 5181-5183; DOI: 10.1016/ s0040-4039(02)00972-3.

10. B. A. Bohn, Introduction to Flavonoids, Harwood Academic, Amsterdam 1998.

11. R. L. Lankey and P. T. Anastas, Life-cycle approaches for assessing green chemistry technologies, Ind. Eng. Chem. Res. 41 (2002) 4498-4502; DOI: 10.1021/ie0108191.

12. P. T. Anastas and J. C. Warner, Green Chemistry: Theory and Practice, Oxford University Press, New York 1998.

13. B. S. Dawane, Y. B. Vibhute, S. G. Konda and M. R. Mali, Synthesis of some new 3-(substituted phenyl)-5-(9-anthryl)-2-pyrazolines, 1-phenyl-3-(substituted phenyl)-5-(9-anthryl)-2-pyrazolines and 2-(9-anthryl)-4-(substituted phenyl)-1,5-benzothiazepines as antibacterial agents, Asian J. Chem. 20 (2008) 4199-4204.

14. J. Chen, S. K. Spear, J. G. Huddleston and R. D. Rogers, Polyethylene glycol and solutions of polyethylene glycol as green reaction, Green Chem. 7 (2005) 64-82; DOI: 10.1039/b413546f.

15. Vogel's Text book of Practical Organic Chemistry, 5th ed., Longman, London 1989.

16. B. S. Dawane and Y. B. Vibhute, Synthesis of some new hydroxyl acetophenones by using iodine and iodic acid, J. Ind. Chem. Soc. 77 (2000) 299.

17. M. H. Bahar and B. K. Sabata, Formylation of some heterocycles: An application of Vilsmeier-Haack reaction. Ind. J. Chem. 20B (1981) 328-329.

18. D. Shrinivasan, N. Sangeetha, T. Suresh and P. Lakshmanaperumalsamy, Antimicrobial activity of certain Indian medicinal plants used in folkloric medicine, J. Ethnopharmacol. 74 (2001) 217-220; DOI: $10.1016 /$ s0378-8741(00)00345-7. 
B. S. Dawane et al.: An improved procedure for synthesis of some new 1,3-diaryl-2-propen-1-ones using PEG-400 as a recyclable solvent and their antimicrobial evaluation, Acta Pharm. 59 (2009) 473-482.

\section{Poboljšan postupak sinteze nekih novih 1,3-diaril-2-propen-1-ona koristeći PEG-400 kao reciklirajuće otapalo i njihovo antimikrobno vrednovanje}

BHASKAR S. DAWANE, SHANKARAIAH G. KONDA, BASEER M. SHAIKH i RAGHUNATH B. BHOSALE

Opisana je jednostavna i pogodna metoda sinteze novih hetero 1,3-diaril-2-propen-1-ona (kalkona) koristeći poli(etilenglikol) (PEG-400) kao alternativno otapalo. Reakcija je jednoznačna, a uporaba hlapljivih organskih otapala je smanjena. Iskorištenja na produktima su visoka, a reakcijska vremena kraća. Svi sintetizirani spojevi testirani su na antimikrobno djelovanje na nekoliko patogenih mikroorganizama.

Ključne riječi: 1,3-diaril-2-propen-1-oni, PEG-400, antimikrobno djelovanje

Organic Research Laboratory, Department of Chemistry, Yeshwant Mahavidyalaya, Nanded-431602 (M.S) India 